\title{
Local polarization and isothermal local equilibrium in relativistic heavy ion collisions
}

\author{
F. Becattini, M. Buzzegoli, and A. Palermo \\ Università di Firenze and INFN Sezione di Firenze, \\ Via G. Sansone 1, I-50019 Sesto Fiorentino (Florence), Italy \\ G. Inghirami \\ GSI Helmholtzzentrum für Schwerionenforschung GmbH, \\ Planckstr. 1, 64291 Darmstadt, Germany \\ I. Karpenko \\ Faculty of Nuclear Sciences and Physical Engineering, \\ Czech Technical University in Prague, \\ Břehová 7, 11519 Prague 1, Czech Republic
}

\begin{abstract}
We show that the inclusion of a recently found additional term of the spin polarization vector at local equilibrium which is linear in the symmetrized gradients of the velocity field, and the assumption of hadron production at constant temperature restore the quantitative agreement between hydrodynamic model predictions and local polarization measurements in relativistic heavy ion collisions at $\sqrt{s_{\mathrm{NN}}}=200 \mathrm{GeV}$. The longitudinal component of the spin polarization vector turns out to be very sensitive to the temperature value, with a good fit around $155 \mathrm{MeV}$. The implications of this finding are discussed.
\end{abstract}

Introduction - Spin polarization in a relativistic fluid has been observed in the Quark Gluon Plasma (QGP) formed in relativistic heavy ion collisions [1, 2. From a theory standpoint, the quantitative tool to calculate the expected polarization has mostly been a local equilibrium formula relating the mean spin vector of a particle with four-momentum $p$ to the thermal vorticity at the leading order [3]. For a spin $1 / 2$ particle this reads:

$$
S_{\varpi}^{\mu}(p)=-\frac{1}{8 m} \epsilon^{\mu \rho \sigma \tau} p_{\tau} \frac{\int_{\Sigma} \mathrm{d} \Sigma \cdot p n_{F}\left(1-n_{F}\right) \varpi_{\rho \sigma}}{\int_{\Sigma} \mathrm{d} \Sigma \cdot p n_{F}},
$$

where thermal vorticity is defined as the anti-symmetric derivative of the four-temperature field:

$$
\varpi_{\mu \nu}=-\frac{1}{2}\left(\partial_{\mu} \beta_{\nu}-\partial_{\nu} \beta_{\mu}\right) .
$$

The four-temperature vector is related to the fourvelocity $u$ and the comoving temperature $T$ by $\beta^{\mu}=$ $u^{\mu} / T$. In the (1), $n_{F}$ is the Fermi-Dirac phase-space distribution function: $n_{F}=\{\exp [\beta \cdot p-q \mu / T]+1\}^{-1}$.

The measured global spin polarization of $\Lambda$ hyperons, integrated over all momenta, turns out to be in quantitative agreement with the predictions of the formula (1), the thermal vorticity field being provided by hydrodynamic simulations 44 and by other models 9 11. However, the predicted spin polarization as a function of momentum, the so-called local polarization, disagrees with the measurements. Particularly, the sign of the longitudinal component of the spin polarization vector and the trend of the component perpendicular to the reaction plane as a function of the azimuthal angle are opposite to the model predictions [12].

For the QGP, the formula (1) is applicable to the final hadrons, provided that $\Sigma$ is identified with the hadroniza- tion 3D hypersurface or, more rigorously, the hypersurface where the system ceases to be a fluid at local thermodynamic equilibrium. The failure of (1) in reproducing local polarization stimulated much work in the field. While it has become clear that hadronic decays cannot be responsible for the discrepancies [13, 14, investigations have been undertaken on the impact of dissipative corrections [15 19], of hadronic interactions [20, 21], on kinetic equilibration 22 26] and on the possible role of the spin tensor and an associated spin potential [16, 2730. One may wonder whether the inclusion of quadratic and higher order terms in thermal vorticity would fix the discrepancies. However, thermal vorticity - which is adimensional in natural units - is definitely less than 1 over the decoupling hypersurface (see fig. 1) and subleading corrections are not expected to cure the problem.

Spin polarization from thermal shear - Lately, it has been observed that, at the linear order in the gradients, there is an unexpected additional contribution to spin polarization vector at local equilibrium [31, 32. In the derivation of ref. [31], for a spin $1 / 2$ particle, this reads:

$$
S_{\xi}^{\mu}(p)=-\frac{1}{4 m} \epsilon^{\mu \rho \sigma \tau} \frac{p_{\tau} p^{\lambda}}{\varepsilon} \frac{\int_{\Sigma} \mathrm{d} \Sigma \cdot p n_{F}\left(1-n_{F}\right) \hat{t}_{\rho} \xi_{\sigma \lambda}}{\int_{\Sigma} \mathrm{d} \Sigma \cdot p n_{F}}
$$

where $\varepsilon=\sqrt{m^{2}+\mathbf{p}^{2}}, \hat{t}$ is the time direction in the QGP or center-of-mass frame, and $\xi$ is the symmetric derivative of the four-temperature, defined as thermal shear tensor:

$$
\xi_{\mu \nu}=\frac{1}{2}\left(\partial_{\mu} \beta_{\nu}+\partial_{\nu} \beta_{\mu}\right) .
$$

This additional term is also a purely local equilibrium term, i.e. non-dissipative, and provides a contribution 
to the spin which is comparable to (1) (see fig. 11. It is obtained 31] by expanding the thermodynamic field $\beta$ in the Local thermodynamic Equilibrium (LE) density operator:

$$
\widehat{\rho}_{\mathrm{LE}}=\frac{1}{Z_{\mathrm{LE}}} \exp \left[-\int_{\Sigma} \mathrm{d} \Sigma_{\mu}\left(\widehat{T}^{\mu \nu} \beta_{\nu}-\widehat{j}^{\mu} \zeta\right)\right]
$$

from the point $x$ where the Wigner function $W^{+}(x, p)$, entering the general expression of the spin polarization vector:

$$
S^{\mu}(p)=\frac{1}{2} \frac{\int_{\Sigma} \mathrm{d} \Sigma \cdot p \operatorname{tr}\left[\gamma^{\mu} \gamma^{5} W^{+}(x, p)\right]}{\int_{\Sigma} \mathrm{d} \Sigma \cdot p \operatorname{tr}\left[W^{+}(x, p)\right]},
$$

must be evaluated. In the eq. (5), $\widehat{T}$ is the symmetrized Belinfante stress-energy tensor operator, $\widehat{j}$ is a conserved current and $\zeta T$ its associated chemical potential. It is worth dwelling into some mathematical details behind the formulae (1) and (3). When evaluating the mean value of a local quantum operator (such as the Wigner function) at some space-time point $x$ with the LE density operator $(5)$, i.e. $O(x)=\operatorname{Tr}\left(\widehat{\rho}_{\mathrm{LE}} \widehat{O}(x)\right)$, in the hydrodynamic limit of slowly varying $\beta$ and $\zeta$ fields, one can obtain a good approximation by Taylor expanding the fields at the same point $x$ and replacing a truncated expansion at some order in the exponent of (5) as well as in the partition function $Z$ [33, 34]. For instance, at the first order:

$$
\beta_{\nu}(y) \simeq \beta_{\nu}(x)+\partial_{\lambda} \beta_{\nu}(x)(y-x)^{\lambda}
$$

and replacing into the $(5)$, with $\zeta=0$ which is a good approximation for the purpose of this work:

$$
\begin{aligned}
& \widehat{\rho}_{\mathrm{LE}} \simeq \frac{1}{Z_{\mathrm{LE}}} \exp \left[-\beta_{\nu}(x) \widehat{P}^{\nu}+\right. \\
& \left.-\partial_{\lambda} \beta_{\nu}(x) \int_{\Sigma} \mathrm{d} \Sigma_{\mu}(y)(y-x)^{\lambda} \widehat{T}^{\mu \nu}(y)\right] \\
& =\frac{1}{Z_{\mathrm{LE}}} \exp \left[-\beta(x) \cdot \widehat{P}-\left(\frac{1}{T} \partial_{\lambda} u_{\nu}(x)+u_{\nu} \partial_{\lambda}(1 / T)\right)\right. \\
& \left.\times \int_{\Sigma} \mathrm{d} \Sigma_{\mu}(y)(y-x)^{\lambda} \widehat{T}^{\mu \nu}(y)\right]
\end{aligned}
$$

In the above equation, $\widehat{P}^{\nu}$ is the four-momentum operator, $\beta_{\nu}(x) \widehat{P}^{\nu}$ is the usual global thermodynamic equilibrium exponent with constant four-temperature equal to $\beta(x)$ and the second term in the exponent is the leading gradient correction; also, the contributions from temperature gradient and velocity gradient have been split for later use. Retaining the zeroth order of the above expansion corresponds to the so-called perfect fluid approximation; going to higher orders implies including nondissipative quantum corrections in the local equilibrium calculations. Since the gradient of $\beta$ supposedly gives rise to a small correction to the leading term $\beta_{\nu}(x) \widehat{P}^{\nu}$,
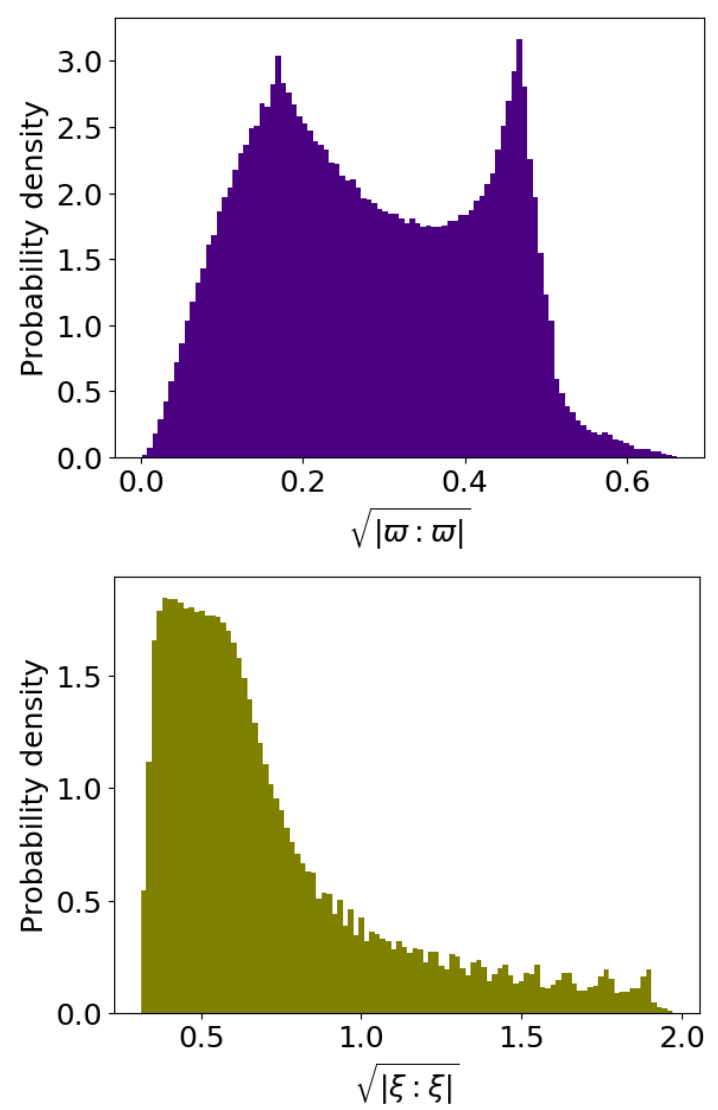

FIG. 1. Distributions of the magnitudes of the thermal voriticity $\sqrt{|\varpi: \varpi|}$ (top) and thermal shear $\sqrt{|\xi: \xi|}$ (bottom) at the decoupling hypersurface for a temperature of $165 \mathrm{MeV}$ and impact parameter $9.2 \mathrm{fm}$ at $\sqrt{s}_{\mathrm{NN}}=200 \mathrm{GeV}$.

one can handle it as a perturbation and apply linear response theory to obtain an approximation of the mean value which is linear in the gradient. Starting from the general formula (6) and splitting $\partial \beta$ in the eq. (7) into an anti-symmetric and a symmetric part eventually yield the two terms (1) and (3) respectively 31. It is also worth pointing out that the latter term stems from a correlator with a non-conserved integral operator [31, which explains the appearance of one particular vector $\hat{t}$ in the formula (3); this vector can be interpreted as a sort of mean normal vector perpendicular to the hypersurface $\Sigma$.

Gradient expansion for relativistic nuclear collisions at very high energy - In the formulae (1), (3) and (6), the hypersurface $\Sigma$ should be the decoupling hypersurface in order to be applicable to the quasi-free hadronic effective fields and, at the same time, to be close to local thermodynamic equilibrium. The question may arise whether the formulae above are the actual best approximations at the leading order in the gradients for the conditions of a relativistic nuclear collision at very high energy. In fact, since the decoupling expectedly occurs at constant temperature, (temperature is the only effective 
intensive variable as chemical potentials are negligible), the approximation can be improved. Indeed, if $\Sigma$ is an isothermal hypersurface, the constant temperature $T$ in $\beta^{\mu}=(1 / T) u^{\mu}$ can be taken out of the integral of the LE density operator:

$$
\widehat{\rho}_{\mathrm{LE}}=\frac{1}{Z_{\mathrm{LE}}} \exp \left[-\frac{1}{T} \int_{\Sigma} \mathrm{d} \Sigma_{\mu} \widehat{T}^{\mu \nu} u_{\nu}\right]
$$

and one can expand in a Taylor series only the fourvelocity $u$, which is not constant over $\Sigma$. Hence, the approximation (7) is replaced with:

$$
\begin{aligned}
\widehat{\rho}_{\mathrm{LE}} \simeq & \frac{1}{Z_{\mathrm{LE}}} \exp \left[-\beta_{\nu}(x) \widehat{P}^{\nu}+\right. \\
& \left.-\frac{1}{T} \partial_{\lambda} u_{\nu}(x) \int_{\Sigma} \mathrm{d} \Sigma_{\mu}(y)(y-x)^{\lambda} \widehat{T}^{\mu \nu}(y)\right] .
\end{aligned}
$$

Comparing the equations, it can be seen that the term proportional to the gradient of temperature in (7) disappeared in the (9). However, it should be emphasized that the temperature gradient $\partial T$ itself does not vanish and it is indeed perpendicular to the hypersurface $T=$ const. Consequently, the term proportional to the gradient of temperature in the expansion (7) is non-vanishing even if the hypersurface $\Sigma$ is $T=$ const and it eventually contributes to both the spin polarization vector expressions in eqs. (1) and (3). In fact, the inclusion of such a term obtained by expanding in full space-time a function which is constant over the hypersurface, makes the first-order approximation of the actual LE (5) a worse one, as it introduces a term which would eventually be cancelled in the full Taylor series. In other words, it is not necessary, neither is it a good approximation, to expand in space-time a function which is constant over some submanifold of space-time (like a 3D hypersurface) if one has to integrate over that sub-manifold. In conclusion, the approximation (9) is more accurate than the (7) for the density operator (5) if $\Sigma$ is a $T=$ const hypersurface. With the (approximated) density operator (9), it is straightforward to obtain the spin polarization vector in the linear response theory; comparing the (7) with the (9), all we have to do is to make the effective replacement:

$$
\partial \beta \rightarrow \frac{1}{T_{\mathrm{dec}}} \partial u
$$

where $T_{\mathrm{dec}}$ is the constant decoupling temperature, in both the equation (1) and (3). Particularly, the spin polarization vector of an emitted spin $1 / 2$ baryon becomes:

$$
\begin{aligned}
& S_{\mathrm{ILE}}^{\mu}(p)= \\
& -\epsilon^{\mu \rho \sigma \tau} p_{\tau} \frac{\int_{\Sigma} \mathrm{d} \Sigma \cdot p n_{F}\left(1-n_{F}\right)\left[\omega_{\rho \sigma}+2 \hat{t}_{\rho} \frac{p^{\lambda}}{\varepsilon} \Xi_{\lambda \sigma}\right]}{8 m T_{\operatorname{dec}} \int_{\Sigma} \mathrm{d} \Sigma \cdot p n_{F}}
\end{aligned}
$$

where ILE stands for isothermal local equilibrium,

$$
\omega_{\rho \sigma}=\frac{1}{2}\left(\partial_{\sigma} u_{\rho}-\partial_{\rho} u_{\sigma}\right)
$$

is the kinematic vorticity and:

$$
\Xi_{\rho \sigma}=\frac{1}{2}\left(\partial_{\sigma} u_{\rho}+\partial_{\rho} u_{\sigma}\right)
$$

is the kinematic shear tensor. It should be stressed that the equation $10 p$ is not equal to the sum of $S_{\varpi}^{\mu}(p)$ and $S_{\xi}^{\mu}(p)$ integrated over the $T=$ const hypersurface, what is confirmed by numerical computation (see fig. 3). Therefore, the equation $(10)$ is the best approximation of the spin polarization vector of a spin $1 / 2$ baryon, at local equilibrium and at linear order in the gradients of the thermodynamic fields for an isothermal decoupling hypersurface. This equation upgrades the original (1) and we are going to show that it is able to restore the agreement between the hydrodynamic model and the data.

Analysis of $A u-A u$ collisions at $\sqrt{s_{\mathrm{NN}}}=200 \mathrm{GeV}$ To compare the predictions of the hydrodynamic model with typical initial conditions with the polarization data, we have used two different $3+1 \mathrm{D}$ viscous hydrodynamic codes in the Israel-Stewart formulation: vHLLE 35 and ECHO-QGP [36, 37]. The parameters defining the initial conditions have been set to reproduce charged particle multiplicity distribution in pseudo-rapidity as well their elliptic flow and directed flow in $\mathrm{Au}-\mathrm{Au}$ collisions at $\sqrt{s_{\mathrm{NN}}}=200 \mathrm{GeV}$.

In order to match the experimental conditions of the local polarization measurements of $\Lambda$ hyperons [38, we set the same centrality range in our hydrodynamic simulations, corresponding to $20-60 \%$ central $\mathrm{Au}-\mathrm{Au}$ collisions. vHLLE simulations have been initialized with averaged entropy density profile from the Monte Carlo Glauber model, generated by GLISSANDO v.2.702 code [39]; ECHO-QGP has been initialized with optical Glauber initial conditions by using the same method as in ref. [40, with a fixed impact parameter $b$ set to $9.2 \mathrm{fm}$.

In figure 2 we show the components of the rest-frame polarization vector $\mathbf{P}=2 \mathbf{S}_{*}$ along the angular momentum $P_{J}$ and along the beam direction $P_{z}$ (for the description of the QGP conventional reference frame, see [41]) as a function of the transverse momentum of the $\Lambda$ hyperon for rapidity $y=0$, from vHLLE calculation. The upper panels show the predictions of the formula (1), and the lower panels the predictions of the new term (3), at a decoupling temperature $T_{\mathrm{dec}}=165 \mathrm{MeV}$. The two contributions are comparable in magnitude and, most importantly, the new term provides a local polarization in qualitative agreement with the data [38, 42, both for the $P_{J}$ and the $P_{z}$ components, and in agreement with a very recent analysis 43] of the thermal shear contribution. The two terms are added up and the result shown in the upper panels of the figure 3 It can be seen that, although the model predictions are somewhat closer to the experimental findings, there is still a consistent discrepancy: a basically uniform $P_{J} 42$ and still the wrong sign of $P_{z}$ 38. Finally, by using the formula (10), based on isothermal local equilibrium, we obtain polarization 

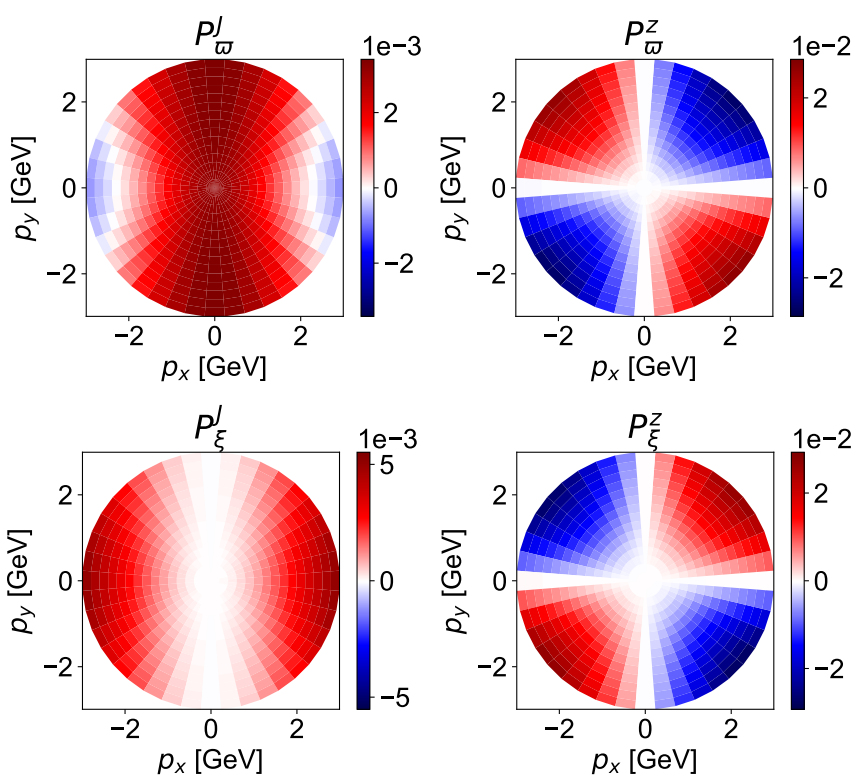

FIG. 2. $\Lambda$ polarization components at mid-rapidity as a function of its transverse momentum $\left(p_{x}, p_{y}\right)$, computed with vHLLE for 20-60\% Au-Au collisions at $\sqrt{s_{\mathrm{NN}}}=200 \mathrm{GeV}$. Upper panel: polarization induced by thermal vorticity $\varpi$, lower panel: polarization induced by thermal shear $\xi$.
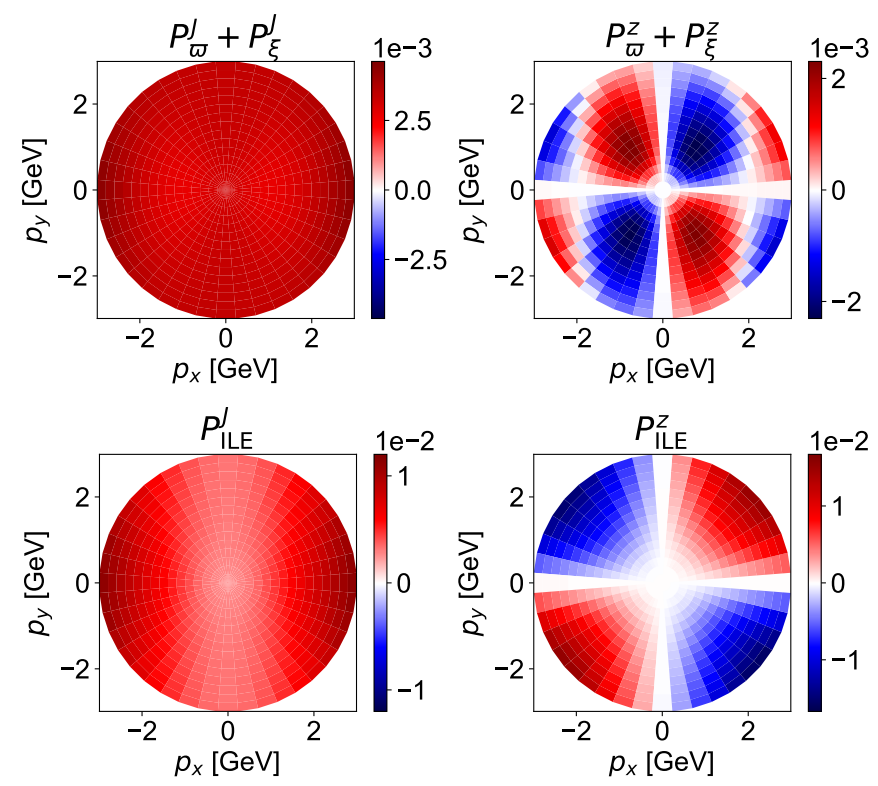

FIG. 3. Same as Fig. 2, with the upper panels showing the sum of $S_{\varpi}^{\mu}$ and $S_{\xi}^{\mu}$ from equations (1) and (3); the lower panels show the predictions of eq. 10.

distributions, shown in the lower panels of fig. 3. which are in an agreement with the measurements, with the right sign of $P_{z}$ and the qualitatively correct $P_{J}(\phi)$ dependence. These findings are confirmed by a corresponding analysis made with the ECHO-QGP code and shown in fig. 4 .
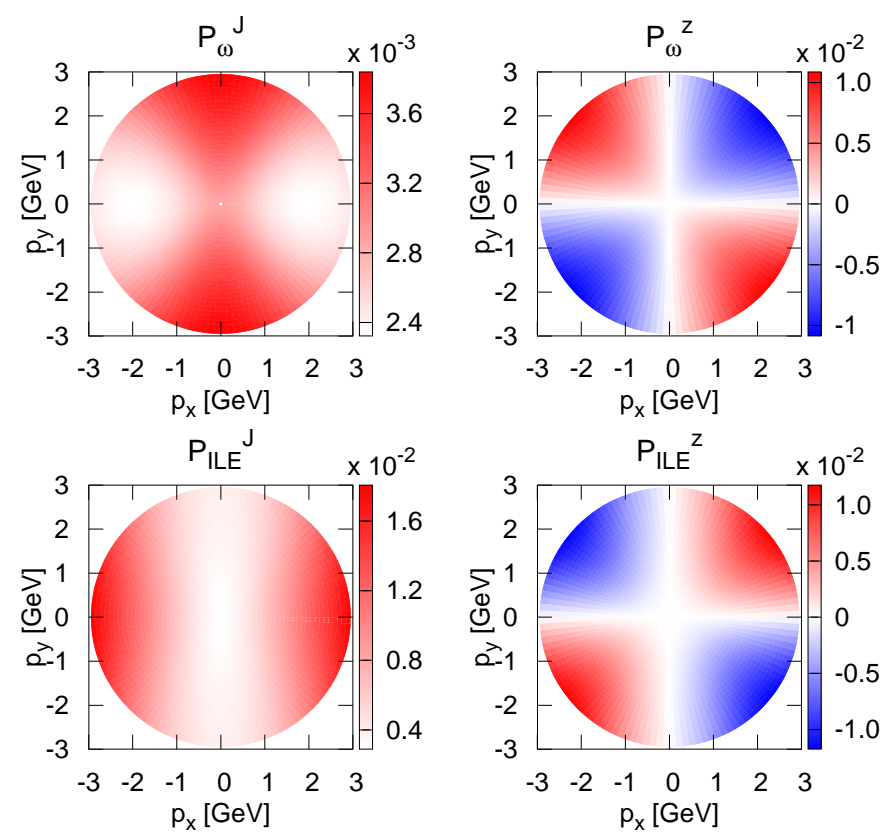

FIG. 4. $\Lambda$ polarization components at mid-rapidity as a function of its transverse momentum $\left(p_{x}, p_{y}\right)$, computed with ECHO-QGP. Upper panel: contribution from the first term in equation 10 induced by $\omega / T$. Lower panel: full prediction of equation $(10)$.

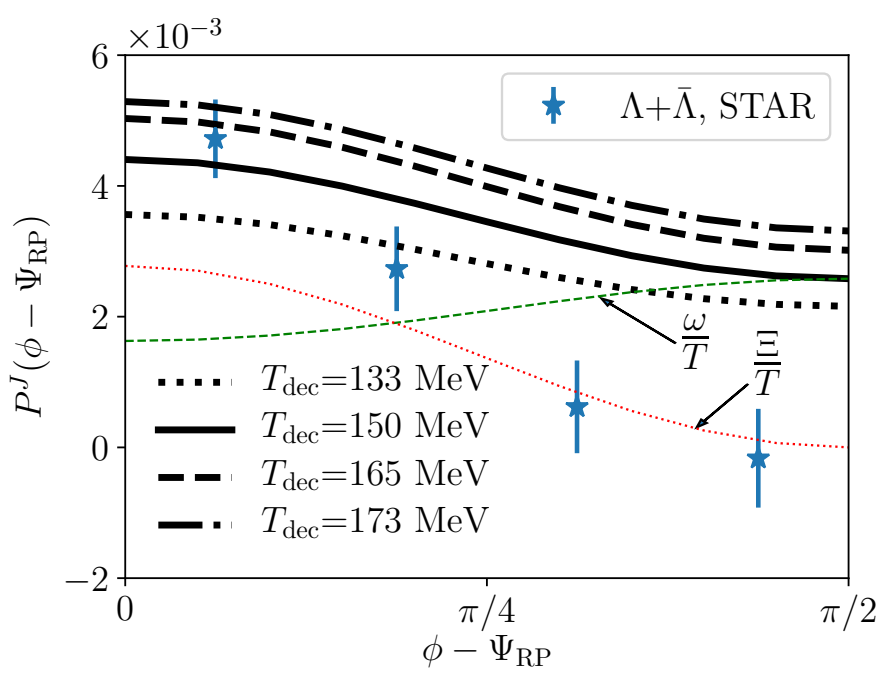

FIG. 5. $\Lambda$ polarization component along the global angular momentum, as a function of the azimuthal angle $\phi$, computed with vHLLE for $20-60 \% \mathrm{Au}-\mathrm{Au}$ collisions at $\sqrt{s_{\mathrm{NN}}}=$ $200 \mathrm{GeV}$. Experimental data points are taken from 42 .

Finally, we have compared the data with the predictions of the eq. 10 at four different decoupling temperatures in figs. 5 and 6 by integrating the $p_{T}$ spectrum of the $\Lambda$ in the same range as in the data, that is $0.5-6$ $\mathrm{GeV}$. It can be seen that the longitudinal component $P_{z}$ is very sensitive to the decoupling temperature, and it is in very good agreement with the data, for $T_{\mathrm{dec}}$ value 


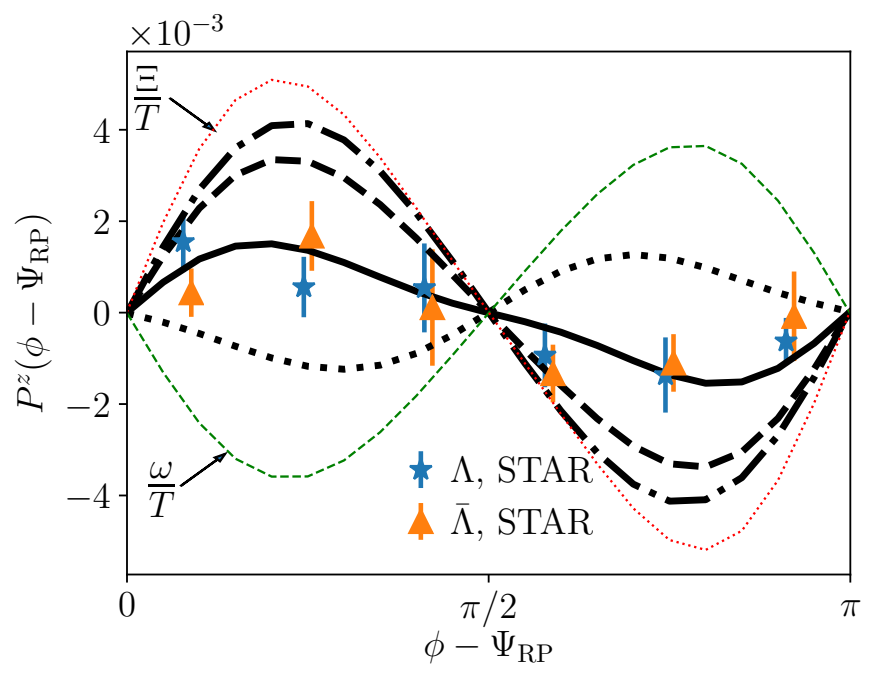

FIG. 6. $\Lambda$ polarization component along the beam direction, as a function of the azimuthal angle $\phi$, computed with vHLLE for $20-60 \% \mathrm{Au}-\mathrm{Au}$ collisions at $\sqrt{s_{\mathrm{NN}}}=200 \mathrm{GeV}$. Experimental data points are taken from 38] and conversion from $\left\langle\cos \theta_{p}^{*}\right\rangle$ to $P_{H}$ is performed using $\alpha_{\mathrm{H}}=0.732$ [44]. Error bars represent the sum of statistical and systematic uncertainties. Line styles correspond to different decoupling temperatures as in Fig. 5

around 150-160 MeV; for temperatures below around 135 $\mathrm{MeV}$, the sign of the longitudinal polarization flips. The $P_{J}$ component is now predicted to have a maximal value on the reaction plane, in agreement with the data, however with a milder descent as a function of the azimuthal angle; also, it is less sensitive to $T_{\mathrm{dec}}$. We also note that the global polarization resulting from the integration of $P_{J}$ is still in a reasonably good agreement with previous calculations. Also shown, in both figures, are the contributions from the kinematic vorticity $\omega$ (thin dashed line) and the kinematic shear $\Xi$ (thin smaller dashed line), at the decoupling temperature of $150 \mathrm{MeV}$. It can be seen in figure 6 that the latter is crucial to flip the sign of $P_{z}$ and restore the agreement with the data, while the vorticity term alone would give the wrong sign, as already remarked in ref. [6].

Discussion, conclusions and outlook - The recently found additional shear term and the realization of the constancy of $T_{\mathrm{dec}}$ are the two key ingredients to reproduce the local polarization and the $P_{J}$ and $P_{z}$ patterns. This finding is thus a striking confirmation of the local equilibrium picture or, in perhaps more suggestive words, the quasi-ideal fluid paradigm of the QGP, even in the spin sector. Dissipative corrections to spin polarization may play a role, but they appear not to be decisive. The standard hydrodynamic picture with the initial conditions obtained by fitting radial spectra, elliptic and directed flow, works very well for the local polarization too. Another strong indication from this finding is that, at very high energy, the QGP hadronizes in space-time at constant $T_{\mathrm{dec}}$ to a more accurate level than one could have imagined. Indeed, its sensitivity to the gradients of the thermodynamic fields, makes spin the ideal probe to investigate the space-time details of hadron formation. Furthermore, as we have shown, the longitudinal spin polarization turns out to be very sensitive to the decoupling temperature, the causes of which deserve to be studied in detail. Looking ahead to future investigations, it is certainly important to compare the predictions of the formula 10 as a function of transverse momentum and rapidity besides azimuthal angle. At lower energy, where the chemical potentials are relevant, one can expect a decoupling hypersurface different from the simple $T=$ const, and this will require a reconsideration of the 107 in order to obtain accurate predictions.

Acknowledgments - G. Inghirami acknowledges funding by the Deutsche Forschungsgemeinschaft (DFG, German Research Foundation) - Project number 315477589 - TRR 211. M.B. is supported by the Florence University fellowship Effetti quantistici nei fluidi relativistici. I.K. acknowledges support by the project Centre of Advanced Applied Sciences with number CZ.02.1.01/0.0/0.0/16019/0000778, which is co-financed by the European Union, and support by the Ministry of Education, Youth and Sports of the Czech Republic under grant "International Mobility of Researchers - MSCA IF IV at CTU in Prague" No. CZ.02.2.69/0.0/0.0/20_079/0017983. Computational resources were supplied by the project "eInfrastruktura CZ" (e-INFRA LM2018140) provided within the program Projects of Large Research, Development and Innovations Infrastructures.

[1] L. Adamczyk et al. (STAR), Nature 548, 62 (2017), arXiv:1701.06657 [nucl-ex]

[2] J. Adam et al. (STAR), Phys. Rev. C 98, 014910 (2018), arXiv:1805.04400 [nucl-ex]

[3] F. Becattini, V. Chandra, L. Del Zanna, and E. Grossi, Annals Phys. 338, 32 (2013), arXiv:1303.3431 [nucl-th],

[4] I. Karpenko and F. Becattini, Eur. Phys. J. C 77, 213 (2017), arXiv:1610.04717 [nucl-th].

[5] Y. Xie, D. Wang, and L. P. Csernai, Phys. Rev. C 95, 031901 (2017), arXiv:1703.03770 [nucl-th].

[6] H.-Z. Wu, L.-G. Pang, X.-G. Huang, and Q. Wang, Phys. Rev. Research. 1, 033058 (2019), arXiv:1906.09385 [nuclth]

[7] Y. B. Ivanov, (2020), arXiv:2012.07597 [nucl-th]

[8] B. Fu, K. Xu, X.-G. Huang, and H. Song, Phys. Rev. C 103, 024903 (2021), arXiv:2011.03740 [nucl-th]

[9] H. Li, L.-G. Pang, Q. Wang, and X.-L. Xia, Phys. Rev. C 96, 054908 (2017), arXiv:1704.01507 [nucl-th],

[10] D.-X. Wei, W.-T. Deng, and X.-G. Huang, Phys. Rev. C 99, 014905 (2019), arXiv:1810.00151 [nucl-th].

[11] O. Vitiuk, L. V. Bravina, and E. E. Zabrodin, Phys. Lett. B 803, 135298 (2020), arXiv:1910.06292 [hep-ph],

[12] F. Becattini and M. A. Lisa, Ann. Rev. Nucl. Part. Sci. 70, 395 (2020), arXiv:2003.03640 [nucl-ex], 
[13] F. Becattini, G. Cao, and E. Speranza, Eur. Phys. J. C 79, 741 (2019), arXiv:1905.03123 [nucl-th].

[14] X.-L. Xia, H. Li, X.-G. Huang, and H. Z. Huang, Phys. Rev. C 100, 014913 (2019), arXiv:1905.03120 [nucl-th],

[15] K. Hattori, M. Hongo, X.-G. Huang, M. Matsuo, and H. Taya, Phys. Lett. B 795, 100 (2019), arXiv:1901.06615 [hep-th]

[16] N. Weickgenannt, E. Speranza, X.-l. Sheng, Q. Wang, and D. H. Rischke, (2020), arXiv:2005.01506 [hep-ph]

[17] S. Bhadury, W. Florkowski, A. Jaiswal, A. Kumar, and R. Ryblewski, Phys. Lett. B 814, 136096 (2021), arXiv:2002.03937 [hep-ph].

[18] S. Bhadury, W. Florkowski, A. Jaiswal, A. Kumar, and R. Ryblewski, Phys. Rev. D 103, 014030 (2021), arXiv:2008.10976 [nucl-th].

[19] S. Shi, C. Gale, and S. Jeon, (2020), arXiv:2008.08618 [nucl-th]

[20] L. P. Csernai, J. I. Kapusta, and T. Welle, Phys. Rev. C 99, 021901 (2019), arXiv:1807.11521 [nucl-th]

[21] M. G. L. Nogueira-Santos and C. C. Barros, Int. J. Mod. Phys. E 29, 2050013 (2020), arXiv:2005.08943 [hep-ph],

[22] J.-H. Gao and Z.-T. Liang, Phys. Rev. D 100, 056021 (2019), arXiv:1902.06510 [hep-ph].

[23] J.-j. Zhang, R.-h. Fang, Q. Wang, and X.-N. Wang, Phys. Rev. C 100, 064904 (2019), arXiv:1904.09152 [nucl-th].

[24] S. Y. F. Liu, Y. Sun, and C. M. Ko, Phys. Rev. Lett. 125, 062301 (2020), arXiv:1910.06774 [nucl-th].

[25] S. Li and H.-U. Yee, Phys. Rev. D 100, 056022 (2019), arXiv:1905.10463 [hep-ph]

[26] J. I. Kapusta, E. Rrapaj, and S. Rudaz, Phys. Rev. C 101, 031901 (2020), arXiv:1910.12759 [nucl-th].

[27] W. Florkowski, B. Friman, A. Jaiswal, and E. Speranza, Phys. Rev. C 97, 041901 (2018), arXiv:1705.00587 [nuclth],

[28] W. Florkowski, A. Kumar, R. Ryblewski, and R. Singh, Phys. Rev. C 99, 044910 (2019), arXiv:1901.09655 [hepph]

[29] W. Florkowski, A. Kumar, R. Ryblewski, and
A. Mazeliauskas, Phys. Rev. C 100, 054907 (2019) arXiv:1904.00002 [nucl-th]

[30] A. D. Gallegos, U. Gürsoy, and A. Yarom, (2021), arXiv:2101.04759 [hep-th],

[31] F. Becattini, M. Buzzegoli, and A. Palermo, (2021), arXiv:2103.10917 [nucl-th], to appear in Physics Letters $\mathrm{B}$.

[32] S. Y. F. Liu and Y. Yin, preprint (2021), arXiv:2103.09200 [hep-ph].

[33] F. Becattini, L. Bucciantini, E. Grossi, and L. Tinti, Eur. Phys. J. C 75, 191 (2015), arXiv:1403.6265 [hep-th]

[34] F. Becattini (2020) arXiv:2004.04050 [hep-th]

[35] I. Karpenko, P. Huovinen, and M. Bleicher, Comput. Phys. Commun. 185, 3016 (2014), arXiv:1312.4160 [nuclth]

[36] L. Del Zanna, V. Chandra, G. Inghirami, V. Rolando, A. Beraudo, A. De Pace, G. Pagliara, A. Drago, and F. Becattini, Eur. Phys. J. C 73, 2524 (2013) arXiv:1305.7052 [nucl-th].

[37] G. Inghirami, L. Del Zanna, A. Beraudo, M. H. Moghaddam, F. Becattini, and M. Bleicher, Eur. Phys. J. C 76, 659 (2016), arXiv:1609.03042 [hep-ph]

[38] J. Adam et al. (STAR), Phys. Rev. Lett. 123, 132301 (2019), arXiv:1905.11917 [nucl-ex].

[39] M. Rybczynski, G. Stefanek, W. Broniowski, and P. Bozek, Comput. Phys. Commun. 185, 1759 (2014), arXiv:1310.5475 [nucl-th].

[40] F. Becattini, G. Inghirami, V. Rolando, A. Beraudo, L. Del Zanna, A. De Pace, M. Nardi, G. Pagliara, and V. Chandra, Eur. Phys. J. C 75, 406 (2015), [Erratum: Eur.Phys.J.C 78, 354 (2018)], arXiv:1501.04468 [nucl-th],

[41] F. Becattini and I. Karpenko, Phys. Rev. Lett. 120, 012302 (2018), arXiv:1707.07984 [nucl-th]

[42] T. Niida (STAR), Nucl. Phys. A 982, 511 (2019), arXiv:1808.10482 [nucl-ex]

[43] B. Fu, S. Y. F. Liu, L. Pang, H. Song, and Y. Yin, (2021), arXiv:2103.10403 [hep-ph].

[44] P. Zyla et al. (Particle Data Group), PTEP 2020, 083C01 (2020) 